\title{
Theoretical Aspects of Science with Radioactive Nuclear Beams*
}

\author{
Jacek Dobaczewski ${ }^{1-3}$ and Witold Nazarewicz ${ }^{1,3,4}$ \\ ${ }^{1}$ Department of Physics, University of Tennessee, Knoxville, TN 37996, U.S.A. \\ ${ }^{2}$ Joint Institute for Heavy Ion Research, Oak Ridge National Laboratory \\ P.O. Box 2008, Oak Ridge, TN 37831, U.S.A. \\ ${ }^{3}$ Institute of Theoretical Physics, Warsaw University \\ Hoża 69, PL-00681, Warsaw, Poland \\ ${ }^{4}$ Physics Division, Oak Ridge National Laboratory \\ P.O. Box 2008, Oak Ridge, TN 37831, U.S.A.
}

\begin{abstract}
Physics of radioactive nuclear beams is one of the main frontiers of nuclear science today. Experimentally, thanks to technological developments, we are on the verge of invading the territory of extreme $N / Z$ ratios in an unprecedented way. Theoretically, nuclear exotica represent a formidable challenge for the nuclear many-body theories and their power to predict nuclear properties in nuclear terra incognita. It is important to remember that the lesson learned by going to the limits of the nuclear binding is also important for "normal" nuclei from the neighborhood of the beta stability valley. And, of course, radioactive nuclei are crucial astrophysically; they pave the highway along which the nuclear material is transported up in the proton and neutron numbers during the complicated synthesis process in stars.
\end{abstract}

\section{INTRODUCTION}

There are only 263 stable nuclei; they are surrounded by radioactive ones. Some of the unstable nuclei are long-lived and can be found on Earth, some are man-made (actually, as many as $\sim 2,200$ nuclei have been produced in nuclear laboratories), and several thousand nuclei are the yet-unexplored exotic species. The decay characteristics of most radioactive nuclei are determined by the beta decay, i.e., by weak interactions. For heavier nuclei, where the electromagnetic interaction plays a more important role, other decay channels, such as emission of alpha particles or spontaneous fission, dominate. Moving away from stable nuclei by adding either protons or neutrons, one finally reaches the particle drip lines. The nuclei beyond the drip lines are unbound to nucleon emission; that is, for those systems the strong interaction is unable to bind $A$ nucleons as one nucleus.

*Will be published in Theme Issue on Science with Beams of Radioactive Nuclei, Philosophical Transactions, ed. by W. Gelletly 
So, the territory of exotic nuclei is enormous. The uncharted regions of the $(N, Z)$ plane contain information that can answer many questions of fundamental importance for science: How many protons and neutrons can be clustered together by the strong interaction to form a bound nucleus? What are the proton and neutron magic numbers of the exotic nuclei? What are the properties of very short-lived exotic nuclei with extreme neutron-to-proton ratio $N / Z$ ? What is the effective nucleon-nucleon interaction in the nucleus having a very large neutron excess? There are also related questions in the field of nuclear astrophysics. Since radioactive nuclei are produced in many astrophysical sites, knowledge of their properties is crucial to the understanding of the underlying processes.

Nuclear life far from stability is different from that around the stability line; the promised access to completely new combinations of proton and neutron numbers offers prospects for new structural phenomena. The unique structural factor is the weak binding; hence closeness to the particle continuum. The main objective of this paper is to discuss some of the theoretical challenges and opportunities of research with exotic nuclear beams.

The paper is organized as follows. Section M contains a brief review of theoretical developments related to the physics of exotic nuclei. Sections [IT] and IV contain some of the physics issues of the neutron and proton drip lines, respectively, including a discussion on the theoretical uncertainties in determining the particle drip lines. Finally, conclusions are contained in Sec. \.

\section{NEW THEORETICAL ASPECTS OF PHYSICS WITH EXOTIC BEAMS}

From a theoretical point of view, spectroscopy of exotic nuclei offers a unique test of those components of effective interactions that depend on the isospin degrees of freedom. In principle, the effective nucleon-nucleon interaction in heavy nuclei should be obtained by means of the Brückner renormalization which corrects the free interaction for the effects due to the nuclear medium. In practice, however, the effective interaction is approximated by means of some phenomenological density-dependent force with parameters that are usually fitted to stable nuclei and to selected properties of the infinite nuclear matter. Hence, it is by no means obvious that the isotopic trends far from stability predicted by commonly used effective interactions are correct. In the models aiming at such an extrapolation, the important questions asked are: What is the density dependence of the two-body central force [1] [3]? What is the density and radial dependence of the one-body spin-orbit force [4] []? Does the spin-orbit splitting strongly vary with $N / Z$ [8]? What is the form of the pairing interaction in weakly bound nuclei [9 [12]? What is the importance of the effective mass (i.e., the non-locality of the force) for isotopic trends [13]? What is the role of the medium effects (renormalization) and of the core polarization in the nuclear exterior (halo or skin region) where the nucleonic density is small [14] Similar questions are asked in connection with properties of nuclear matter [6, [15] [17], neutron droplets [8], and the physics of the neutron-star crust [18,19].

The radioactive nuclear beams experimentation is expected to expand the range of nuclei known. That is, by going to nuclei with extreme $N / Z$ ratios, one can magnify the isospindependent terms of the effective interaction (which are small in "normal" nuclei). The hope

is that after probing these terms at the limits of extreme isospin, we can later go back to the valley of stability and improve the description of "normal" nuclei. 
But this task is not going to be easy. In many respects, weakly bound nuclei are much more difficult to treat theoretically than well-bound systems. Hence, before tackling the problem of force parametrization at the extremes, one should be sure that the applied theoretical tools of the nuclear many-body problem are appropriate.

As mentioned above, the main theoretical challenge is the correct treatment of the particle continuum. For weakly bound nuclei, the Fermi energy lies very close to zero, and the decay channels must be taken into account explicitly. As a result, many cherished approaches of nuclear theory such as the conventional shell model, the pairing theory, or the macroscopic-microscopic approach must be modified. But there is also a splendid opportunity: the explicit coupling between bound states and continuum, and the presence of low-lying scattering states invite strong interplay and cross-fertilization between nuclear structure and reaction theory. Many methods developed by reaction theory can now be applied to structure aspects of loosely bound systems.

How to extend traditional tools of nuclear theory to account for the scattering of nucleons from bound single-particle orbitals to unbound states? The closeness of particle continuum reverberates in two aspects of the theoretical description. Firstly, the particles forming a bound nuclear state can effortlessly virtually scatter back and forth into the particle continuum phase space. This process must conserve the compactness of the nuclear wave function which remains bound even with such a virtual scattering taken into account. A theoretical description of this kind of effects still remains a virgin territory, although some progress has been made in the analysis of the virtual pair scattering [20,10]. Secondly, nucleons can very easily leave the nucleus altogether and enter the particle continuum through the real scattering. For this, it is enough to slightly shake the nucleus by providing it with a little bit of energy. This is an old problem which, in the context of excited states near or above the particle threshold, has been addressed by the continuum shell model (CSM) [21-28]. In the CSM, the continuum states (decay channels) and bound states are treated on equal footing. Consequently, correlations due to the coupling to resonances, the spatial extension effects in weakly bound states, the structure of resonances, and the structure of particle transfer form factors are properly described by the CSM. So far, most applications of the CSM have been concerned with the situation when there is only one particle occupying the shell-model continuum. This is because the continuum-continuum coupling is difficult to treat [29].

Often, particle continuum is approximated by the quasibound states, i.e., the states resulting from the diagonalization of a finite potential in a large basis 30,31 or by enclosing the finite nuclear potential within an infinite well with walls positioned at a large distance from the nuclear surface [32,33]. More sophisticated methods of discretizing continuum include the Sturmian function expansions and resonant state expansions. Sturmian functions, also known as Weinberg states, form a discrete set of states which behave asymptotically as outgoing waves. They have been used as a basis in the solution of scattering equations, including various applications of the CSM [22,34 37]. The Gamow states are eigenstates of the time-independent Schrödinger equation with complex eigenvalues [38 40]. They have been applied to many problems involving an unbound spectrum [41 43].

In the description of weakly bound systems, pairing interaction plays a unique role (see Section [IIB below). In the BCS or BCS-like methods based on bound and quasibound states (for examples of such calculations see, e.g., Refs. [31,44]), the virtual scattering of nucleonic pairs from bound states to the positive-energy states leads to the presence of 
a "particle gas" surrounding the nucleus [20]. To show it, we decompose the BCS wave function into contributions from bound states $\left(\epsilon_{i}<0\right)$ and quasibound states $\left(\epsilon_{j}>0\right)$ :

$$
\Psi_{B C S}=\prod_{i, \epsilon_{i}<0}\left(U_{i}+V_{i} a_{i}^{\dagger} a_{i}^{\dagger}\right) \prod_{j, \epsilon_{j}>0}\left(U_{j}+V_{j} a_{j}^{\dagger} a_{j}^{\dagger}\right)|0\rangle .
$$

While the bound-state component in Eq. (1) represents the localized wave function, i.e., it decays asymptotically, the second part represents the contribution from quasibound states and leads to non-localized densities with incorrect asymptotic behavior. Indeed, although the nuclear densities eventually vanish at large distances by construction (finite size of the basis, finite size of the box in which calculations are performed), the wave functions of positive-energy states do not decay outside the nuclear volume. As discussed below in Sec. IIIB, this problem is overcome in the Hartree-Fock-Bogolyubov (HFB) method with a realistic pairing interaction in which the coupling of bound states to the particle continuum is correctly taken into account 20,10].

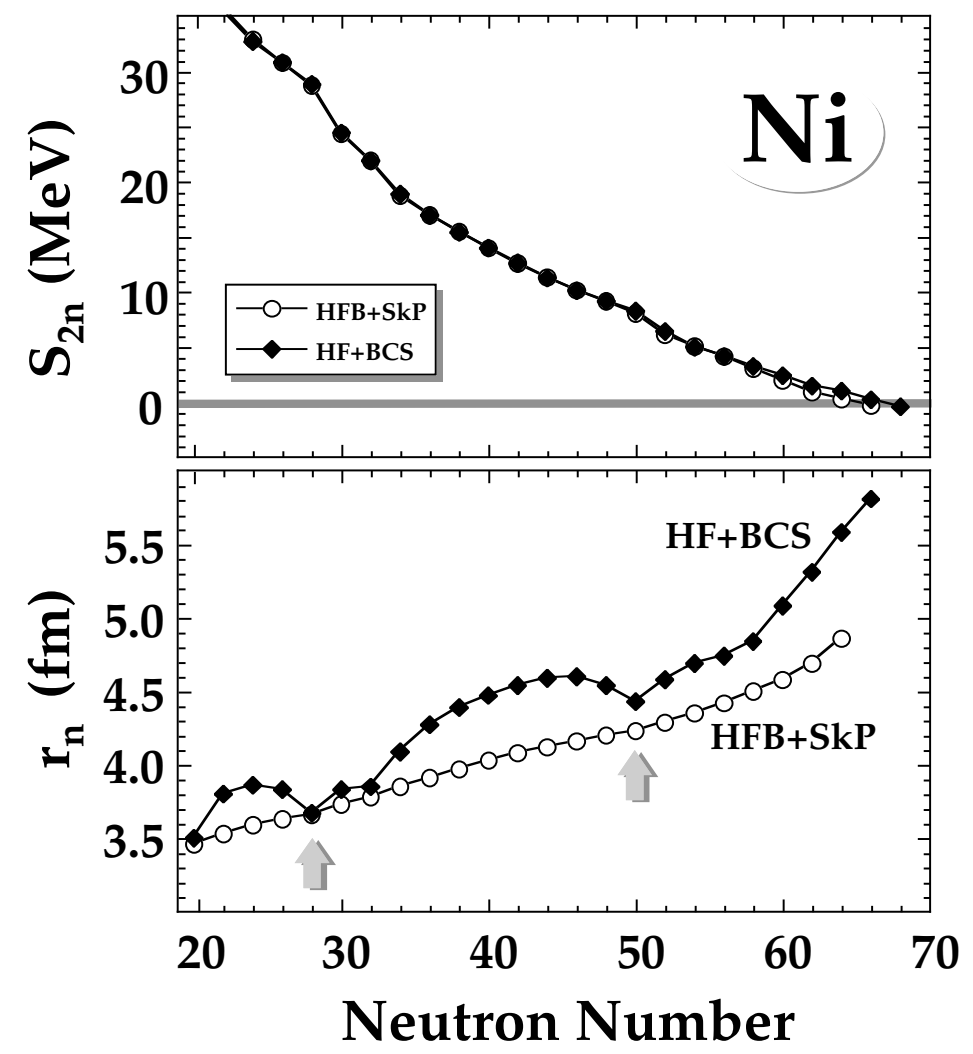

FIG. 1. Top: Two-neutron separation energies for the even-even nickel isotopes predicted in the HFB and HF+BCS calculations with the Skyrme interaction SkP. Bottom: The predicted rms neutron radii. The arrows indicate the neutron subshell closures at $N=28$ and 50. (From Ref. [45.)

To illustrate this point, critical in the context of calculations for drip-line nuclei, Fig. 1 displays theoretical two-neutron separation energies, $S_{2 n}$, and the rms neutron radii for the even-even nickel isotopes obtained in the HFB and HF+BCS calculations. In the HF+BCS variant, the self-consistent pairing gaps obtained from the HFB calculations were used within 
the fixed-gap approximation. As seen in Fig. 1, the values of $S_{2 n}$ obtained in the HFB and $\mathrm{HF}+\mathrm{BCS}$ calculations agree very well; some deviations are seen only for the neutron drip-line systems with $N>60$, where the $\mathrm{HF}+\mathrm{BCS}$ values are slightly lower. However, this excellent agreement does not extend to neutron radii. In the HFB calculations the neutron radii behave very smoothly as a function of $N$. On the other hand, in the HF+BCS model there is a dramatic increase in the neutron radii between magic numbers and for the neutron-rich nuclei resulting from the unphysical occupation of positive-energy quasibound states. The difference between values of radii obtained within the HFB and $\mathrm{HF}+\mathrm{BCS}$ calculations can be as large as $0.8 \mathrm{fm}$ and increases dramatically for weakly bound systems. Other examples illustrating the unphysical effect of the particle gas can be found in Refs. [10,46].

Consequently, for large exotic nuclei, the self-consistent HFB treatment is not a matter of choice, it is a must. The calculations are not easy, especially if the self-consistent symmetries (e.g., spherical symmetry) are broken. Possible strategies for solving the HFB equations include the two-step diagonalization [47], the gradient method in the canonical representation [48], or the state-dependent Hamiltonian method [49]. Some examples of HFB calculations are discussed in the following sections. Other calculations with self-consistent inclusion of pairing and continuum states include: quasiclassical Lagrangian method calculations in the coordinate representation [50 54] and application of the relativistic Hartree-Bogolyubov theory in coordinate space to light nuclei [55.56].

In order to describe excited states, one has to go beyond the mean-field approximation. One method, often used in the description of low-lying collective states in drip-line nuclei, is the continuum random phase approximation (CRPA) based on the single-particle Green function approach in the coordinate representation [57,58]. In particular, the CRPA has been employed extensively to the very low-energy multipole strength in drip-line nuclei [59 62. However, for meaningful predictions of excited states in weakly bound nuclei where pairing is expected to be very important, it is necessary to use the quasiparticle RPA scheme based on the coordinate space HFB formalism. Calculations along these lines can be found in recent Refs. 663,64.

The following sections contain a brief description of selected highlights of the physics of radioactive nuclear beams. It is to be noted that there are many other important topics that have been left out in our discussion (e.g., physics of very light drip-line nuclei, physics of beta decay, reaction aspects involving radioactive ions). A key point is that the variety of exciting new phenomena is one of the driving forces behind research with exotic beams.

\section{PHYSICS OF NEUTRON-RICH NUCLEI: UPPER LIMITS OF THE $N / Z$ RATIO}

Since neutrons do not carry an electric charge and do not repel each other, many neutrons can be added to nuclei starting from the valley of stability. As a result, the "lever arm" separating the neutron drip line from the valley of stability is large and difficult to probe experimentally; except for the lightest nuclei, the bounds of the neutron stability are not known. But it is just for the nuclei with the extreme neutron excess that theory predicts many new and initially unexpected phenomena. In addition to nuclear structure interest, the neutron-rich environment is important for astrophysics and cosmology. 


\section{A. Density distributions}

Neutron-rich nuclei are characterized by spatially extended density distributions which give rise to large nuclear radial moments. Extreme cases are halo nuclei - loosely bound few-body systems with about thrice more neutrons than protons. The halo region is a zone of weak binding in which quantum effects play a critical role in distributing nuclear density in regions not classically allowed.

In the heavier, neutron-rich nuclei, where the concept of mean field is better applicable, the separation into a "core" and "valence nucleons" seems less justified. However, also in these nuclei the weak neutron binding implies the existence of the neutron skin (i.e., a dramatic excess of neutrons at large distances).

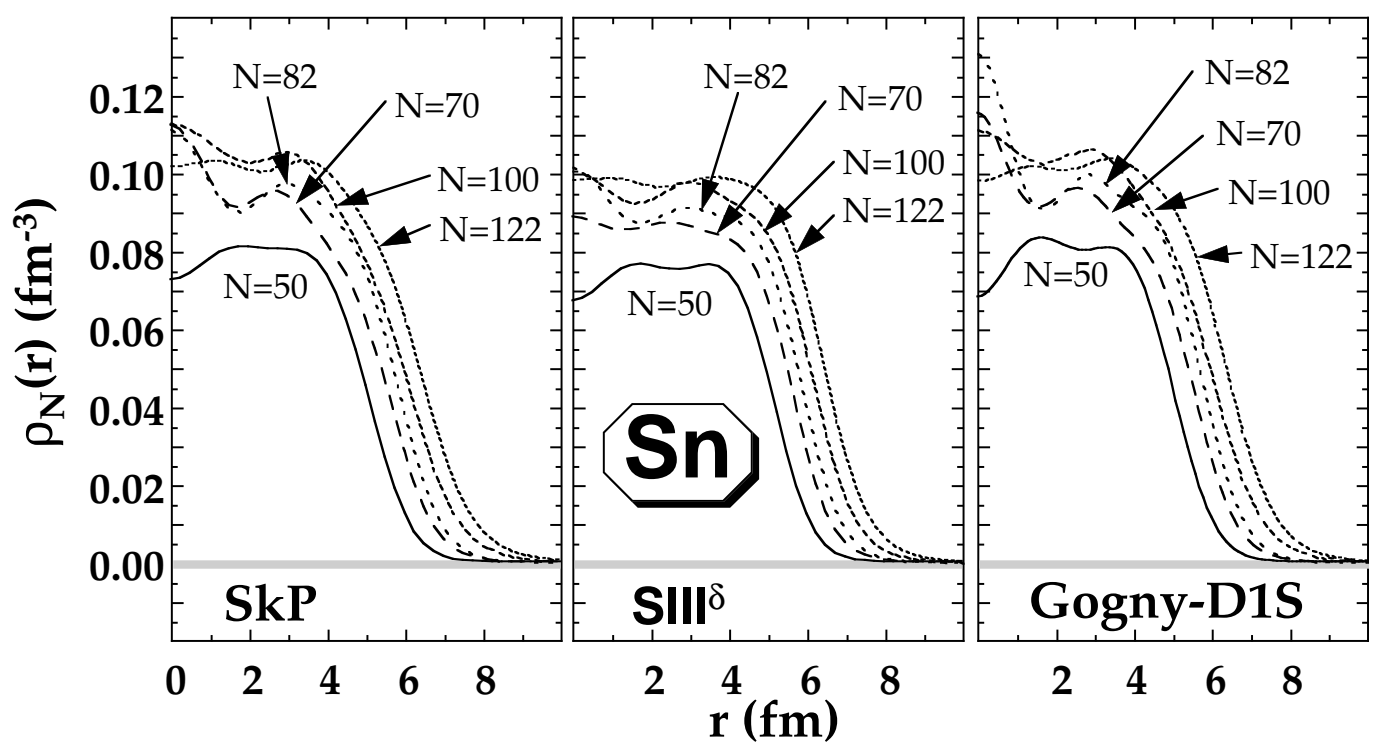

FIG. 2. Self-consistent spherical neutron densities $\rho_{N}(r)$ calculated with the SkP, SIII ${ }^{\delta}$, and D1S interactions for selected tin isotopes across the $\beta$-stability valley. Since proton and neutron densities in the nucleus ${ }^{100} \mathrm{Sn}$ are very similar [65], the deviation from the neutron density at $N=50$ roughly represents the skin effect. (From Ref. [10].)

Figure 2 displays the neutron HFB densities for several tin isotopes across the stability valley, calculated with the effective interactions $\mathrm{SkP}$ [20], $\mathrm{SIII}^{\delta}$ [13], and D1S [66]. The densities obtained with these forces are qualitatively very similar. One can see that adding neutrons results in a simultaneous increase of the central neutron density, and of the density in the surface region. The relative magnitude of the two effects is governed by a balance between the volume and the surface asymmetry energies of effective interactions. Since all three forces considered have been fitted in a similar way to bulk nuclear properties (including the isospin dependence), the resulting balance between the volume and the surface isospin effects is similar. Of course, this does not exclude some differences which are seen when a more detailed comparison is carried out. As will be seen in Sec IIIB below, pair densities depend much stronger on the effective forces. 

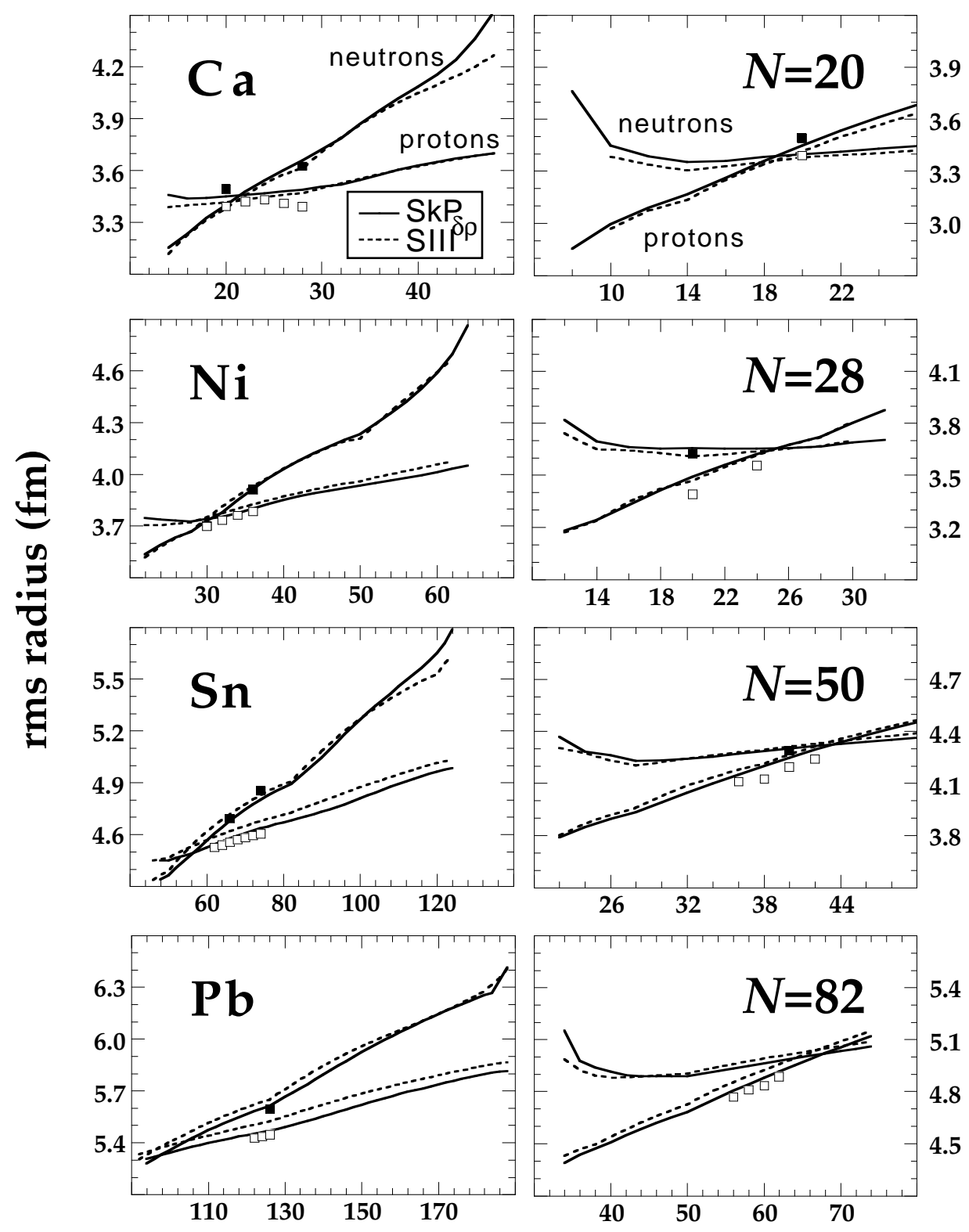

Neutron Number

\section{Proton Number}

FIG. 3. Comparison of experimental (symbols) and calculated (HFB+SkP, solid line; $\mathrm{HFB}+\mathrm{SIII}^{\delta \rho}$, dashed line) proton and neutron rms radii for the semi-magic nuclei with $N$ (or $Z)=20,28$, 50, and 82. The experimental proton radii were extracted from experimental charge radii [67]. The experimental neutron radii were obtained from the analysis of the high-energy proton-nucleus scattering [68]. (From Ref. 45].)

A very interesting aspect of nuclei far from stability is an increase in their radial dimension with decreasing particle separation energy [69]. This effect is especially strong in weakly bound nuclei close to the neutron drip line. Figure 3 presents the comparison of experimental (symbols) and calculated proton and neutron rms radii for the semi-magic isotopes and isotones with $Z($ or $N)=20,28,50$, and 82 . For neutron radii in general, and 
for proton radii in light and medium-mass nuclei, the $\mathrm{HFB}+\mathrm{SkP}$ and $\mathrm{HFB}+\mathrm{SIII}{ }^{\delta \rho}$ models yield very similar predictions. In heavy nuclei $(Z$ or $N=50,82)$ the proton radii in $\mathrm{SkP}$ are lower than in $\mathrm{SIII}^{\delta \rho}$, in closer agreement with experiment. In Fig. 3, the neutron skin is manifested by a rapid increase in neutron rms radii when approaching the two-neutron drip line. This effect is very localized in neutron number; it appears only in a few nuclei in the immediate neighborhood of the neutron drip line.

\section{B. Pairing correlations}

Pairing correlations play a very special role in drip-line nuclei [20,10]. This is seen from the approximate HFB relation between the Fermi level $\lambda$, pairing gap $\Delta$, and the particle separation energy $S \approx-\lambda-\Delta$. At the drip line $S$ is very small and $\lambda+\Delta \sim 0$. Consequently, the single-particle field characterized by $\lambda$ and the pairing field $\Delta$ are equally important. In other words, contrary to the situation encountered close to the line of beta stability, the pairing component of the Hamiltonian can no longer be treated as a residual interaction, i.e., a small perturbation important only in the neighborhood of the Fermi surface.

Surprisingly, very little is known about the pairing channel of the effective nucleonnucleon interaction. In most calculations, the pairing Hamiltonian has been approximated by the state-independent seniority pairing force, or schematic multipole pairing interaction [70]. Such oversimplified forces, usually treated by means of the BCS approximation, perform remarkably well when applied to nuclei in the neighborhood of the stability valley (where, as pointed out above, pairing can be considered as a small correction). As a result, considerable effort was devoted in the past to optimizing the HF part of the interaction, while leaving the pairing component aside.

A detailed discussion of the present status of effective interactions in the particle-particle channel can be found in Ref. [10]. The main questions pertaining to this problem are: What is the microscopic origin of the pairing interaction [71] 75]? What is the role of finite range and the importance of density dependence [76 81]? How can properties of the pairing force be tested experimentally? These questions are of considerable importance not only for nuclear physics but also for nuclear astrophysics and cosmology [18, 82, 83].

Because of strong surface effects, the properties of weakly bound nuclei are perfect laboratories in which to study the density dependence of pairing interactions. As an example of what can be expected far from stability, Fig. 4 displays the neutron HFB local pairing densities $\tilde{\rho}(r)$ calculated for several tin isotopes across the stability valley, and for three different effective interactions: Skyrme interactions $\mathrm{SkP}$ and $\mathrm{SkP}^{\delta}$, and the finite-range Gogny interaction D1S. [The density $\tilde{\rho}(\boldsymbol{r})$ is proportional to the probability of finding the correlated pair of nucleons at point $\boldsymbol{r}$, see Ref. [10] for definitions and discussion.] The pairing densities shown in Fig. 1 nicely reflect different characters of the interactions used. Namely, the contact force $\mathrm{SkP}^{\delta}$ leads to pairing densities that are, in general, largest at the origin and decrease towards the surface; this is characteristic of the volume-type pairing correlations. A different pattern appears for the SkP results, where the density dependence renders the

pairing interaction strongly peaked at the surface. In this case, the pairing densities tend to increase when going from the center of the nucleus towards its surface. A more pronounced dependence on the neutron excess is seen here in the surface region. Near the drip line, the pairing density develops a long tail extending towards large distances. The results ob- 
tained for the finite-range Gogny interaction exhibit features intermediate between surface and volume-type pairing correlations.

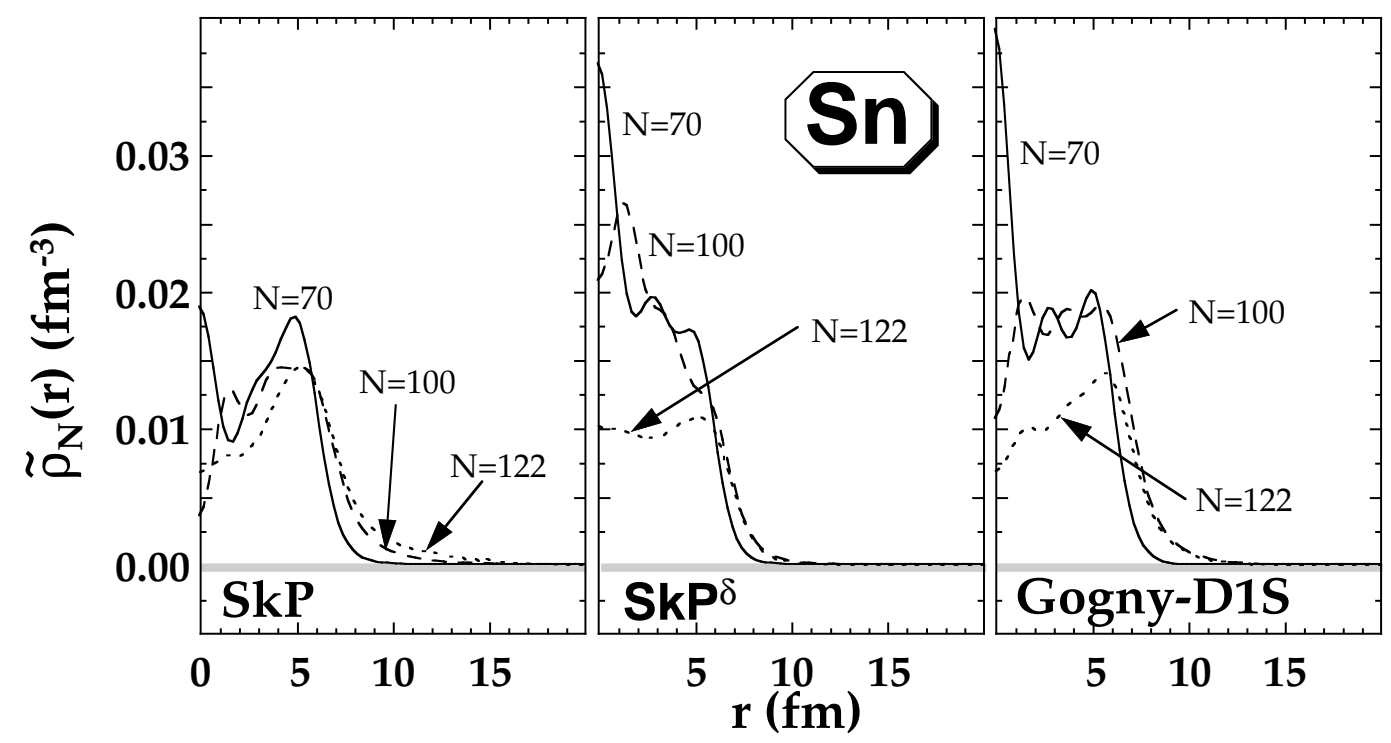

FIG. 4. Self-consistent spherical neutron pairing densities $\tilde{\rho}_{N}(r)$ calculated with the SkP, $\mathrm{SkP}^{\delta}$, and D1S interactions for selected tin isotopes across the $\beta$-stability valley. (From Ref. [10].)

An experimental observable that may probe the character of the pairing field is the pair transfer form factor, directly related to the pairing density $\tilde{\rho}$. The difference in the asymptotic behavior of single-particle density $\rho$ and pair density $\tilde{\rho}$ in a weakly bound system can be probed by comparing the energy dependence of one-particle and pair-transfer cross sections. Such measurements, when performed for both stable and neutron-rich nuclei, can shed some light on the asymptotic properties of the pair densities; hence on the character of the pairing field.

Figure 5 displays the pair transfer form factors $r^{2} \tilde{\rho}(r)$ calculated in ${ }^{120} \mathrm{Sn},{ }^{150} \mathrm{Sn}$, and ${ }^{172} \mathrm{Sn}$ with the $\mathrm{SkP}$ interaction. These pair transfer form factors clearly show that this process has a predominantly surface character. In particular, there is a significant increase in the pair transfer form factors in the outer regions of drip-line nuclei. In ${ }^{120} \mathrm{Sn}$, the form factors vanish around $9 \mathrm{fm}$, while in ${ }^{150} \mathrm{Sn}$ and ${ }^{172} \mathrm{Sn}$ they extend to much larger distances.

\section{Shell structure far from stability and position of the neutron drip line}

The structure of nuclei is expected to change significantly as the limit of nuclear stability is approached in neutron excess. Due to the systematic variation in the spatial distribution of nucleonic densities and the increased importance of the pairing field, the average nucleonic potential is modified when approaching the neutron drip line. The main effect is the increase of the potential diffuseness; the single-particle neutron potential in drip-line nuclei becomes very shallow, and the resulting shell-model spectrum resembles that of a harmonic oscillator with a spin-orbit term and with a weakened $\ell^{2}$ term 65]. This results in a new shell structure characterized by a more uniform distribution of normal-parity orbits and the unique-parity 
intruder orbit which reverts towards its parent shell.

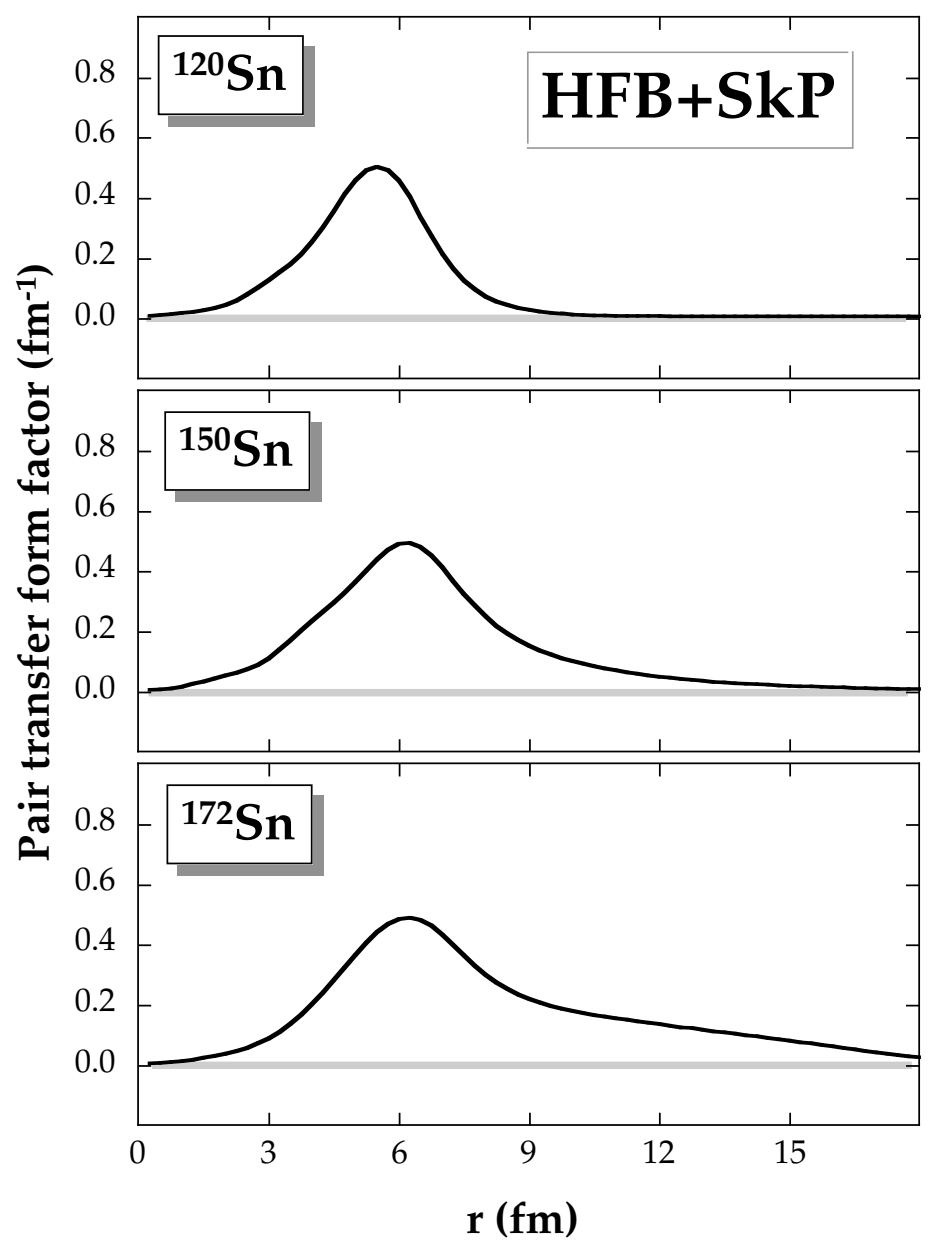

FIG. 5. Pair transfer form factor, $r^{2} \tilde{\rho}(r)$, calculated directly from the HFB pairing density $\tilde{\rho}(r)$. (From Ref. 10.)

The effect of the weakening of shell effects in drip-line nuclei, first mentioned in Ref. [84, was further investigated in Refs. 85, 86, 65, 13, 87, 88,. Quenching of shell effects manifests itself in the behavior of two-neutron separation energies $S_{2 n}$. This is illustrated in Fig. 6 which displays the two-neutron separation energies for the $N=80,82,84$, and 86 spherical even-even isotones calculated in the HFB model with the SkP [20] and SLy4 [89] effective interactions. The large $N=82$ magic gap, clearly seen in the nuclei close to the stability valley and to the proton drip line, gradually closes down when approaching the neutron drip line. This result is independent of the size of the $N=82$ shell gap in stable nuclei, which is slightly underestimated and overestimated by SkP and SLy4 forces, respectively, as compared to experimental data. It can be attributed to two effects: (i) a gradual increase of the neutron surface diffuseness across the stability valley related to an increase of the neutron excess, and (ii) the influence of the continuum, which results in closing the shell gap near the neutron drip line down to zero. 


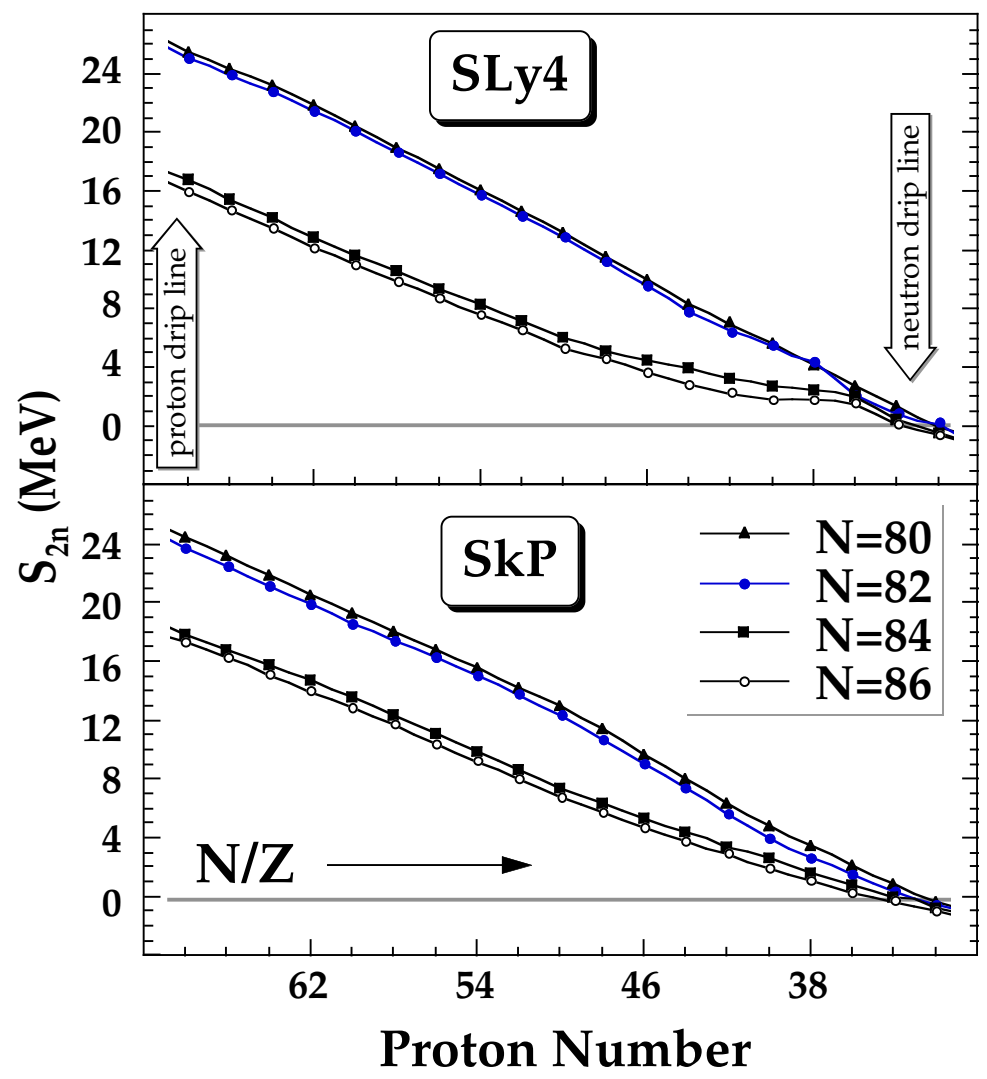

FIG. 6. Two-neutron separation energies for the $N=80,82,84$, and 86 spherical even-even isotones calculated in the $\mathrm{HFB}+\mathrm{SkP}$ and $\mathrm{HFB}+\mathrm{SLy} 4^{\delta}$ models as functions of the proton number. The arrows indicate the proximity of neutron and proton drip lines for small and large proton numbers, respectively.

Predicted behaviour of the two-neutron separation energies depends very much on the effective interaction used. This is illustrated in Fig. 7 which shows the $S_{2 n}$ values calculated in tin isotopes for the Gogny interaction D1S and for four variants of the Skyrme interaction. The Gogny force and the SkP and SLy4 ${ }^{\delta}$ Skyrme forces predict a gradual decrease of the two-neutron separation energies while the older Skyrme forces, $\mathrm{SIII}^{\delta}$ and $\mathrm{SkM}^{\delta}$, give almost constant values followed by a sudden drop at $N=126$. This shows that the $N=126$ shell quenching is not a generic effect.

As seen in Fig. 17, the position of the neutron drip line for the Sn isotopes also depends on the effective interaction used; it varies between $N=118$ (D1S) and $N=126\left(\mathrm{SIII}^{\delta}\right)$. Hence, even if the theoretical method used to calculate nuclear masses is reliable near the drip line (this is not the case for the commonly used macroscopic-microscopic models, see Ref. [31]), the uncertainty due to the largely unknown isospin dependence of the force gives an appreciable theoretical "error bar". 


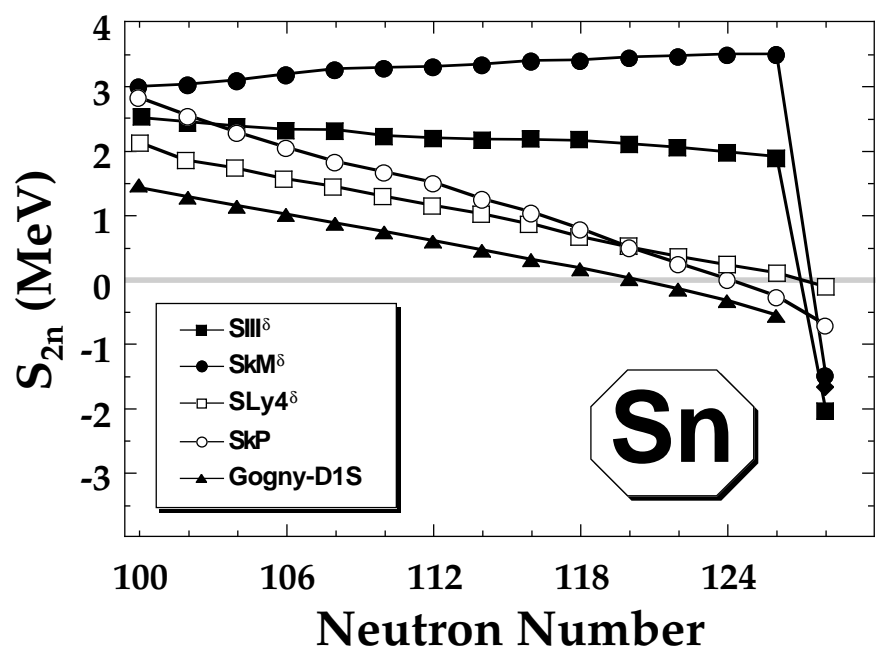

FIG. 7. Two-neutron separation energies $S_{2 n}$ for the Sn isotopes, calculated in the HFB approach with the Skyrme interactions $\mathrm{SIII}^{\delta}, \mathrm{SkM}^{\delta}, \mathrm{SLy}^{\delta}$, and $\mathrm{SkP}$ and with the Gogny-D1S interaction.

Unfortunately, the results presented in Fig. 7 do not tell us much about which of the forces discussed should be the "preferred one" since we are dealing with dramatic extrapolations far beyond the region known experimentally. The comparison with the data can be carried out for nuclei closer to stability, see Ref. 90] for a recent quantitative analysis. A comparison with the experimental two-neutron separation energies is displayed in Fig. 8. As seen, the agreement with data is unsatisfactory for $\mathrm{SIII}^{\delta}$. In particular, the shell-gap sizes at $N=50$, 82 , and 126 are strongly overestimated, and the values and the slopes of $S_{2 n}$ are, in most cases, incorrect. For the $\mathrm{SkM}^{\delta}$ force one obtains similar deficiencies [13. The results shown in Figs. 7 and 8 illustrate a very strong dependence of the two-neutron separation energies on the force parameters. Although older forces, such as SIII and SkM*, can perform well in certain regions of $Z$ and $N$, they do not give a satisfactory global reproduction of the data. On the other hand, a fairly good global agreement obtained with the SkP and SLy $4^{\delta}$ suggests that the improvement is possible, while a still better parametrization would be welcome. Of course, forces which fail in reproducing the behavior with $(N-Z)$ in known nuclei have little chance to perform better when going far from stability. For example, the predicted values of $S_{2 n}$ obtained for the $\mathrm{SIII}^{\delta}$ and $\mathrm{SkM}^{\delta}$ interactions (Fig. (7) followed by a strong shell effect at $N=126$ do not seem very reliable. A detailed analysis of the force-dependence of results may give us valuable information on the relative importance of various force parameters.

The gradual change in shell structure is expected to give rise to new sorts of collective phenomena [31,91]. It is also to be noted that the experimentally observed collapse of magic gaps seen in some neutron-rich light nuclei is conventionally explained in terms of the shape transition to the deformed intruder configuration. Here, spectacular examples are ${ }^{32} \mathrm{Mg}_{20}$ 92,93 and ${ }^{44} \mathrm{~S}_{28}$ [94,95. In both cases, $\mathrm{HF}$ calculations predict the shape transition [96,97. due to the crossing of spherical configuration by intruder states. Such a lowering of the intruder configuration depends on the detailed balance between three components in the total energy: (i) the position of the intruder state at the spherical shape, (ii) the deformation energy gain associated with the deformation-driving orbital, and (iii) the symmetry-restoring 
force exerted by particles in the magic-shell configuration. The first and the third of these elements directly depend on the spherical shell structure and thus can be affected by the hypothetical shell quenching mechanism. However, the appearance of a coexisting configuration cannot, of course, be discussed solely in terms of the spherical shell structure, and it still remains an open and exciting problem.

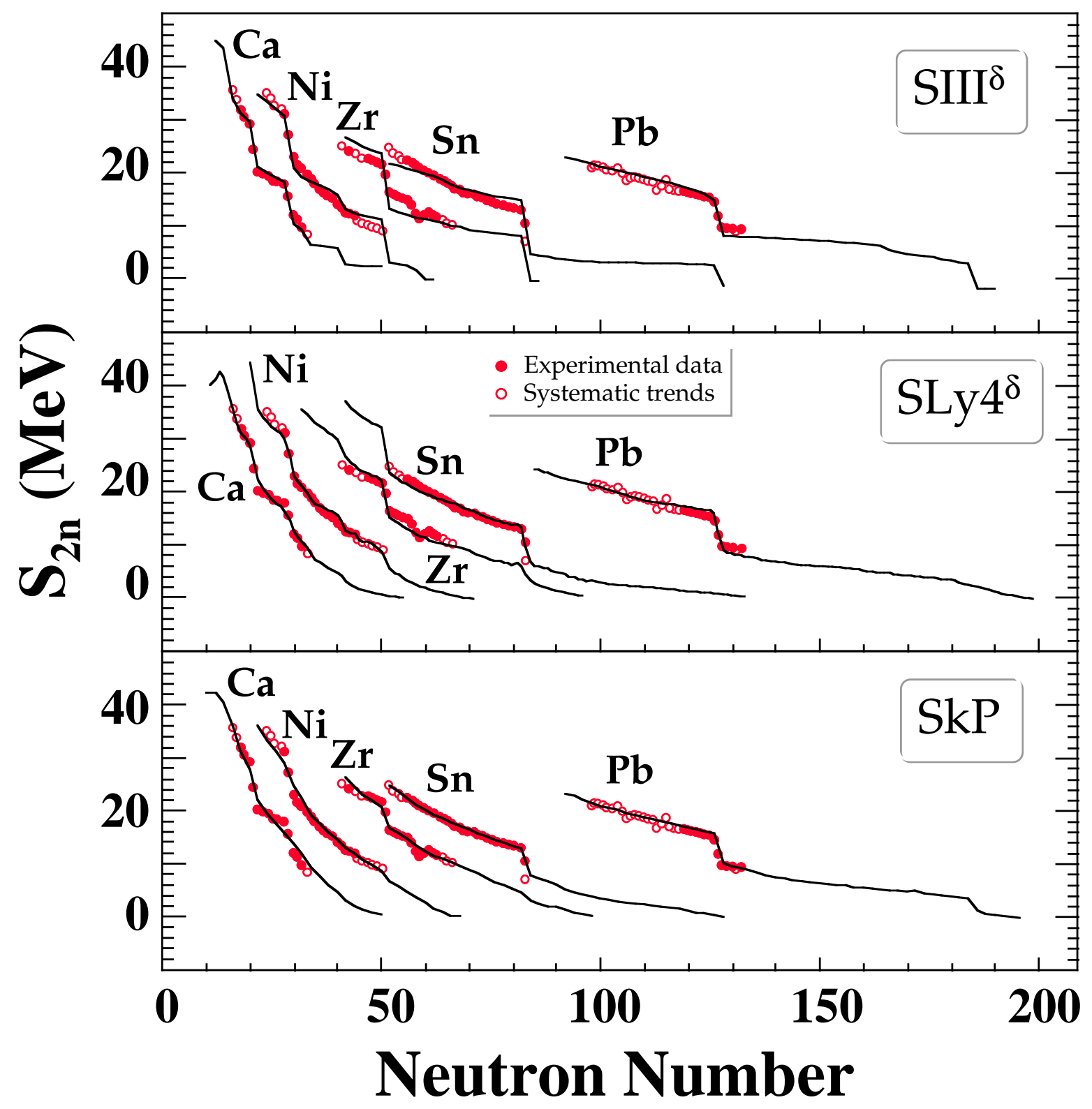

FIG. 8. Two-neutron separation energies, $S_{2 n}$, for proton-magic isotopes. The HFB results with the $\mathrm{SIII}^{\delta}, \mathrm{SLy}^{\delta}$, and $\mathrm{SkP}$ parametrizations (solid lines) are compared with the experimental data (full circles) and systematic trends (open circles) 98].

In the following section we discuss another important aspect of the shell quenching, namely, its consequences for the r-process and the stellar nucleosynthesis. 


\section{Structure of neutron-rich nuclei and the r-process}

The very neutron-rich drip-line nuclei cannot be reached experimentally under present laboratory conditions. On the other hand, these systems are the building blocks of the astrophysical r-process; their separation energies, decay rates, and neutron capture cross sections are the basic quantities determining the results of nuclear reaction network calculations. Consequently, one hopes to learn about properties of very neutron-rich systems by studying the r-process component of the solar-system abundances of heavy elements [99,100,88, 101,102. The recent r-process network calculations [99, 87,,101, based on several mass formulae, indicate that a quenching of the shell effect at $N=82$ is required in order to fill the $A=120$ and 140 r-abundance troughs, in accordance with the results of the $\mathrm{HFB}+\mathrm{SkP}$ model shown in Fig. 6.

In addition to nuclear structure, there are other factors which can influence the r-process abundances, for instance the astrophysical conditions of temperature, neutron density, and the process time scale [103]. The possibility that abundances of r-process elements may be altered by the intense neutrino flux has been discussed in Refs. [104, 105]. According to their calculations, neutrino reactions can be important in breaking through the waiting-point nuclei at $N=50$ and 82, and the r-process abundances in the $A=125$ and 185 regions can be affected by neutrino post-processing effects.

In addition to nuclear masses, another important piece of nuclear structure that determines the path of the r-process is the GT strength. It is usually calculated in the quasiparticle RPA (QRPA) theory [99,106 108. The important development, yet to be done, would consist of performing systematic microscopic QRPA calculations based on the HFB densities. This would guarantee the proper treatment of the particle continuum in the weakly bound nuclei on the r-process path.

\section{E. Deformation of drip-line nuclei}

Neutron halos and heavy, weakly bound neutron-rich nuclei offer an opportunity to study the wealth of phenomena associated with the closeness of the particle threshold: particle emission (ionization to the continuum) and characteristic behavior of cross sections [109,21], existence of soft collective modes and low-lying transition strength [110 113, 61], as well as various other nuclear properties in the sub-threshold regime. We have learned that weakly bound nuclei are different; they have giant sizes, they are diffused, they are strongly superfluid, their shell structure is probably different. But can they be deformed?

The importance of non-spherical intrinsic shapes in halo nuclei has been discussed, especially in the context of a one-neutron halo in ${ }^{11} \mathrm{Be}$. The ground state of ${ }^{11} \mathrm{Be}$ is a $1 / 2^{+}$ state. The low neutron separation energy, $S_{n}=504 \mathrm{keV}$, allows for only one bound excited level $\left(1 / 2^{-}\right.$at $\left.320 \mathrm{keV}\right)$. The halo character of ${ }^{11}$ Be has been confirmed by studies of reaction cross sections [114, and the importance of deformation can be inferred from the large

quadrupole moment of its core ${ }^{10} \mathrm{Be},|Q|=229 \mathrm{mb}$ [115]. The role of deformation in lowering the excitation energy of the $1 / 2^{+}$intruder level in ${ }^{11} \mathrm{Be}$ has been recognized [116,117, but the joint effect of loose binding and deformation has not been considered. (See, however, recent references 118 122].) 
In a recent study [123], the notion of shape deformations in halo nuclei has been addressed by considering the single-particle motion in the axial spheroidal square well. The properties of the deformed single-particle states, especially in the subthreshold region, were analyzed by making the multipole decomposition in the spherical partial waves with well-defined orbital angular momentum. It has been concluded that in the limit of very weak binding, the geometric interpretation of shape deformation is lost. That is, the deformation of the halo is solely determined by the spatial structure of the valence state wave function, independently of the shape of the core. The deformed core merely establishes the quantization axis of the system - important for determining the angular momentum projection on the symmetry axis, $\Lambda$.

Figure 9 shows the contour map of $P_{\ell}$ (probability to find the partial wave $\ell$ in a given Nilsson state $\left.\left[n_{\text {exc }} \Lambda \pi\right]\right)$ for the $\Lambda=0$ orbitals as functions of binding energy and deformation. The structure of the $[10+]$ Nilsson level, originating from the spherical $1 s$ state, is completely dominated by the $\ell=0$ component, even at very large deformations. A rather interesting pattern is seen in the diagram for the $[20+]$ orbital originating from the spherical $1 d$ state. The $\ell=2$ component dominates at low and medium deformations, and the corresponding probability $P_{\ell=2}$ slowly decreases with $\delta$ at large deformations approaching the (constant) asymptotic limit. However, a similar effect, namely the decrease of the $\ell=2$ component, is seen when approaching the zero binding energy threshold. In the language of the perturbation theory [21], this rapid transition comes from the coupling to the low-energy $\ell=0$ continuum. As a consequence of the dominating role of the $s$-wave (and $p$-wave, for negative-parity states), in the limit of weak binding, the total quadrupole deformation of the (core+valence) system depends solely on the geometry of the valence orbital. Namely, it is consistent with a superdeformed shape $(\pi=-, \Lambda=0$ halo), a spherical shape $(\pi=+, \Lambda=0$ halo), or an oblate shape $(\pi=-, \Lambda=1$ halo), regardless of the deformation of the core. In the language of the self-consistent mean-field theory, this result reflects the extreme softness of the system to the quadrupole distortion. Shape deformation is an extremely powerful concept provided that the nuclear surface can be properly defined. However, for very diffused and spatially extended systems, the geometric interpretation of multipole moments and deformations is lost.

The presence of the spatially extended neutron halo gives rise to the low-energy isovector modes. The deformation decoupling of the halo implies that the nuclei close to the neutron drip line are excellent candidates for isovector quadrupole deformations, with different quadrupole deformations for protons and neutrons. An example of such a situation has been predicted in the self-consistent calculations for the neutron-rich sulfur isotopes performed using the Skyrme HF and relativistic mean-field methods [97,124]. When approaching the neutron drip line, the calculated values of quadrupole deformation for neutrons are systematically smaller than those of the proton distribution. Another promising candidate for such effects is the ${ }^{120} \mathrm{Sr}$ nucleus which is presently being studied by the GCM configuration mixing of the Skyrme HF states [125].

The discussion of deformation in halo nuclei applies to systems with very small binding energy and with negligible pairing. How will this scenario be modified in the presence of pairing, and for greater separation energies? We do not know the answer to this question at present; the systematic investigation of the interplay between deformation and pairing in weakly bound neutron-rich nuclei is one of the most exciting avenues of RNB physics. 


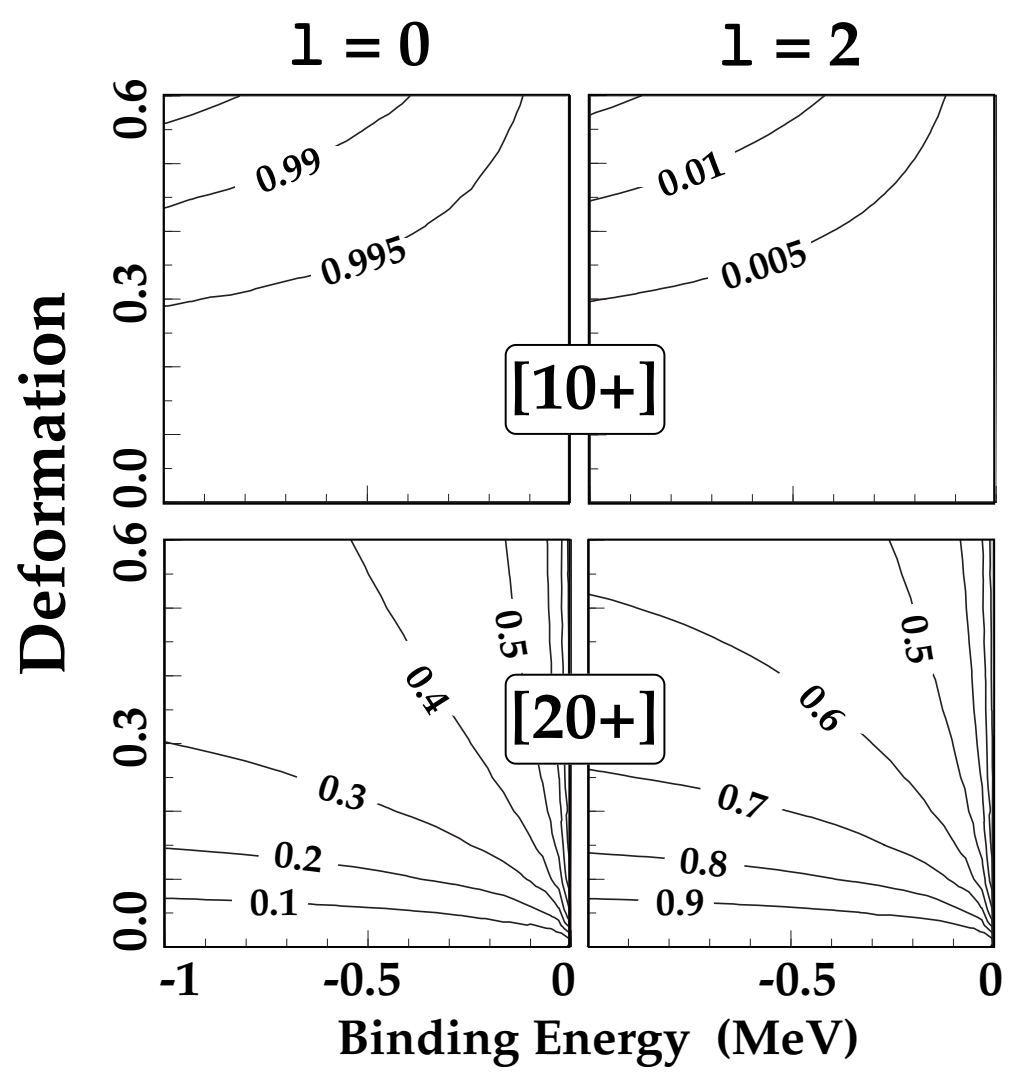

FIG. 9. Contour maps of probabilities $P_{0}$ and $P_{2}$ for the [10+] (top) and [20+] (bottom) Nilsson levels as functions of deformation and binding energy. (From Ref. [123].)

\section{PHYSICS OF PROTON-RICH NUCLEI: AT AND BELOW THE $N / Z=1$ LIMIT}

On the proton-rich side of the valley of stability, physics is different than in nuclei with a large neutron excess. Because of the Coulomb barrier which tends to localize the proton density in the nuclear interior, nuclei beyond the proton drip line are quasibound with respect to proton emission. However, in spite of the stabilizing effect of the Coulomb barrier, the effects associated with the weak binding are also present in proton drip-line nuclei. They are not as dramatic as on the other side of the stability valley, but nevertheless important. For instance, the Thomas-Ehrman shift [126, 127], which is due to changes in the Coulomb energy of the weakly bound proton, can lead to a decrease in the energy differences between analog states by a few hundred keV. This effect is most significant for loosely bound states and for orbitals having low angular momentum [128]. (For the self-consistent calculations of the Thomas-Ehrman shift in the doubly-magic diproton emitter ${ }^{48} \mathrm{Ni}$, see Ref. [46].) Consequently, indiscriminate applications of the nuclear shell-model to nuclei close to and beyond the proton drip line, ignoring the systematic changes in single-particle energies and wave functions due to weak binding, should probably be taken with a grain of salt.

The doubly magic $N=Z=50$ nucleus ${ }^{100} \mathrm{Sn}$ is a paradigm of RNB physics at the protonrich side. Although it was found experimentally three years ago [129, 130], it took more than two years to roughly determine its mass [131], and it will probably take quite a few years to 
find its first excited state. Actually, the question what is this state constitutes an unresolved problem which is a challenge for theoretical predictions.

\section{A. Neutron-proton correlations}

A unique aspect of proton-rich nuclei with $N=Z$ is that neutrons and protons occupy the same shell-model orbitals. Consequently, due to the large spatial overlaps between neutron and proton single-particle wave functions, the proton-rich $N=Z$ nuclei are expected to exhibit unique manifestations of proton-neutron (pn) pairing [132 136].

At present, it is not clear what the specific experimental fingerprints of the pn pairing are, whether the pn correlations are strong enough to form a static pair condensate, and what are their main building blocks [137]. Most of our knowledge about nuclear pairing comes from nuclei with a sizable neutron excess where the isospin $T=1$ neutron-neutron and protonproton pairing dominate. Now, for the first time, there is an experimental opportunity to explore nuclear systems in the vicinity of the $N=Z$ line which have many valence $n p$ pairs; that is, to probe the interplay between the like-particle and pn $(T=0,1)$ pairing channels.

This novel situation calls for the generalization of established theoretical models of nuclear pairing. In spite of several early attempts to extend the independent quasi-particle formalism to incorporate the effect of pn correlations in light nuclei (see Ref. [138] for an early review), no symmetry-unrestricted calculations for np pairing, based on the isospinprojected quasi-particle theory, have been carried out.

So far, the strongest evidence for enhanced pn correlations around the $N=Z$ line comes from the measured binding energies. An additional binding (the so-called Wigner energy) found in these nuclei manifests itself as a spike in the isobaric mass parabola as a function

of $T_{z}=\frac{1}{2}(N-Z)$ (see the review [139] and Refs. quoted therein). The pn correlations are also expected to play a role in beta decay [140 143], deuteron transfer reactions [144, 145], structure of high spins [138, 146, 147], and also in nuclear matter [148,149,82].

The role of the $T=0$ part of the interaction on the presence of binding-energy irregularities near the $N=Z$ has been recognized in Ref. 152. Recent calculations 151 have revealed the rather complex mechanism responsible for the nuclear binding around the $N=Z$ line. In particular, it has been found that the Wigner term cannot be solely explained in terms of correlations between the proton-neutron $J=1, T=0$ (deuteron-like) pairs (see Fig. 10). (For more discussion of this point, see also Ref. [153.)

Recently, the isospin structure of the density matrices and self-consistent mean fields has been discussed [154] in the HFB theory allowing for a consistent microscopic description of pairing correlations in all isospin channels. Theoretically, the pn pairing correlations have been studied by several authors in the HFB framework [155, 156]. However, in Ref. [154 this has been done in the coordinate space allowing for the classification of generic proton-neutron mixing mean fields. The resulting HFB equations have interesting properties. For spherical nuclei, only the $T=1$ and $J=0$ nucleonic pairs are allowed. (The presence of the $T=0$ and $J \neq 0$ pairs would necessarily lead to deformed mean fields.) Consequently, for the spherical symmetry, two cases can be considered. The first one corresponds to pn pairs coupled to $S=0$ and is similar to the standard like-particle pairing. The second one, analogous to triplet pairing with $S=1$, is more interesting because of breaking of the intrinsic parity. Here, 
the HFB solution contains mixtures of proton and neutron states with different parities but with the same value of $j$, e.g., a mixing of the $\nu g_{7 / 2}$ and $\pi f_{7 / 2}$ orbitals.

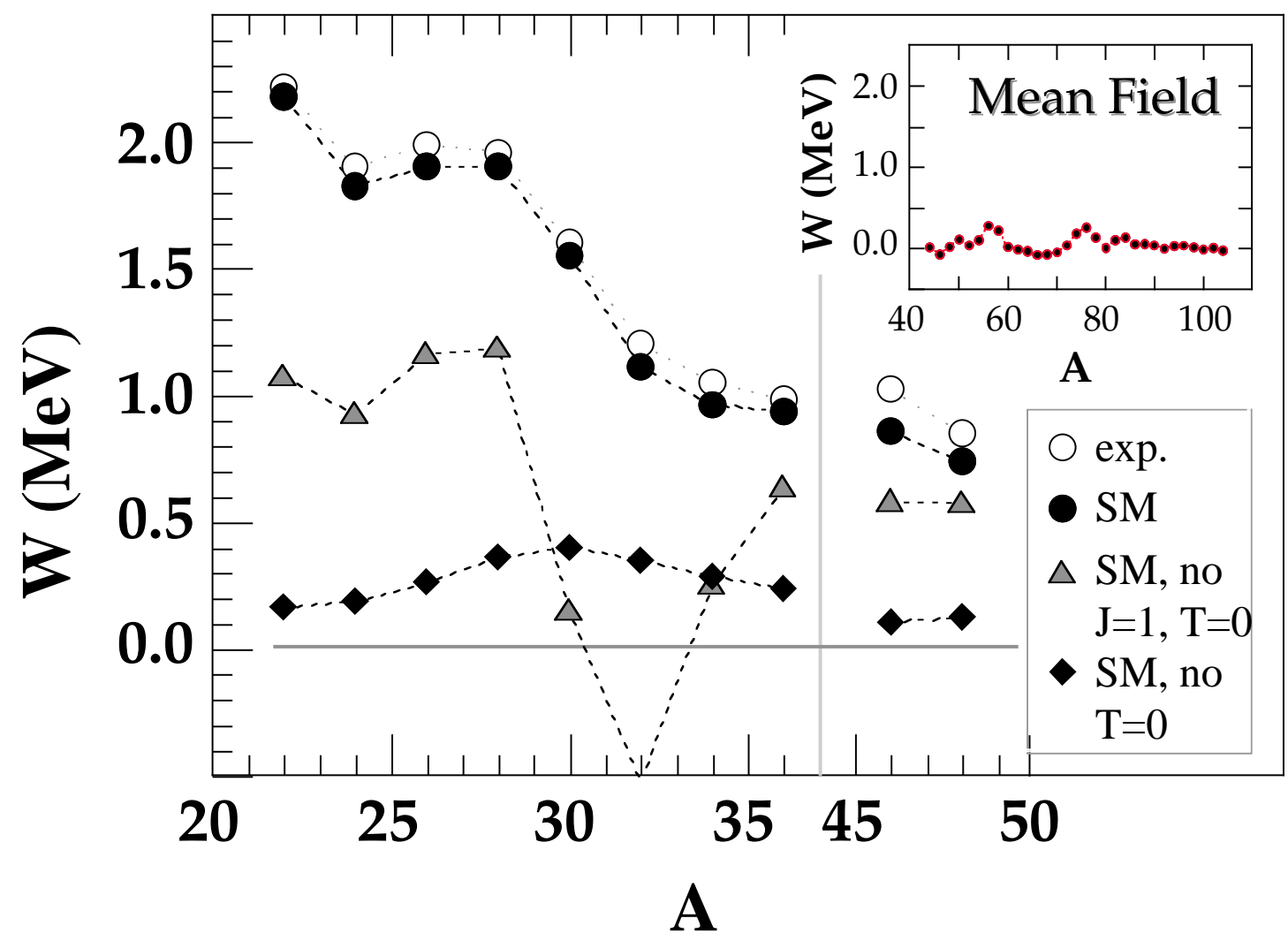

FIG. 10. The strength of the Wigner term, $W$, extracted using binding energies calculated with the $0 \hbar \omega$ shell model. Full shell-model calculations (filled circles) agree very well with experimental data (open circles). The results of shell-model calculations with the $(J=1, T=0)$ two-body matrix elements removed ( $J_{\max }=1$ variant, triangles) and with all $T=0$ matrix elements removed $\left(J_{\max }=7\right.$ variant, diamonds), are also shown. The inset shows the values of $W$ extracted from the ETFSI mass formula [150]. They are practically zero for all nuclei considered. (From Ref. [151].)

\section{B. Proton emitters}

Nuclei beyond the proton drip line are ground-state proton emitters. Initially, the parent nucleus is in a quasistationary state, and the proton emission may be considered as a process where the proton tunnels through the potential barrier. In most cases, the combined Coulomb and centrifugal potentials give rise to barriers which are as large as $\sim 15 \mathrm{MeV}$. Consequently, the associated lifetimes, ranging from $10^{-6} \mathrm{sec}$ to a few seconds, are sufficiently long to obtain a wealth of spectroscopic information. Experimentally, a number of proton emitters have now been discovered in the mass regions $A \sim 110,150$, and 170 (see Refs. 157,158 and references quoted therein). It is anticipated that new regions of protonunstable nuclei will be explored in the near future using radioactive nuclear beams.

The width of the proton resonance can be estimated through the distorted-wave Born approximation (DWBA) [159]. From the decay width one can obtain the half-life of the 
proton emission, $t_{1 / 2}$. The proton resonances are extremely narrow, $\Gamma \sim 10^{-22}-10^{-15} \dot{\mathrm{MeV}}$, hence it is difficult to calculate their widths directly. (It is worth noting that in Ref. [160] an attempt was made to calculate the proton emission width by solving the Schrödinger equation in the complex plane.)

Recently, half-lives of the spherical proton emitters have been calculated in Ref. [161] in the core-plus-proton approach assuming $V_{A p}$ to be a sum of a simple nuclear optical Woods-Saxon potential and the Coulomb potential. Three different methods have been used: DWBA, the modified two-potential approach of Gurvitz [162], and the semi-classical approximation WKB. (Because of its simplicity, the WKB approach has been widely used to study spherical proton emitters [163.)

After computing the barrier penetration factor, the experimental spectroscopic factors $S_{p}^{\exp }$ could be determined as ratios of calculated and measured half-lives [158]. Theoretically, the spectroscopic factor measures the fragmentation of a single-particle orbital $(n \ell j)$. In the BCS theory, it is given by $u_{j}^{2}$, i.e., the probability that the spherical orbital $(n \ell j)$ is empty in the daughter nucleus.

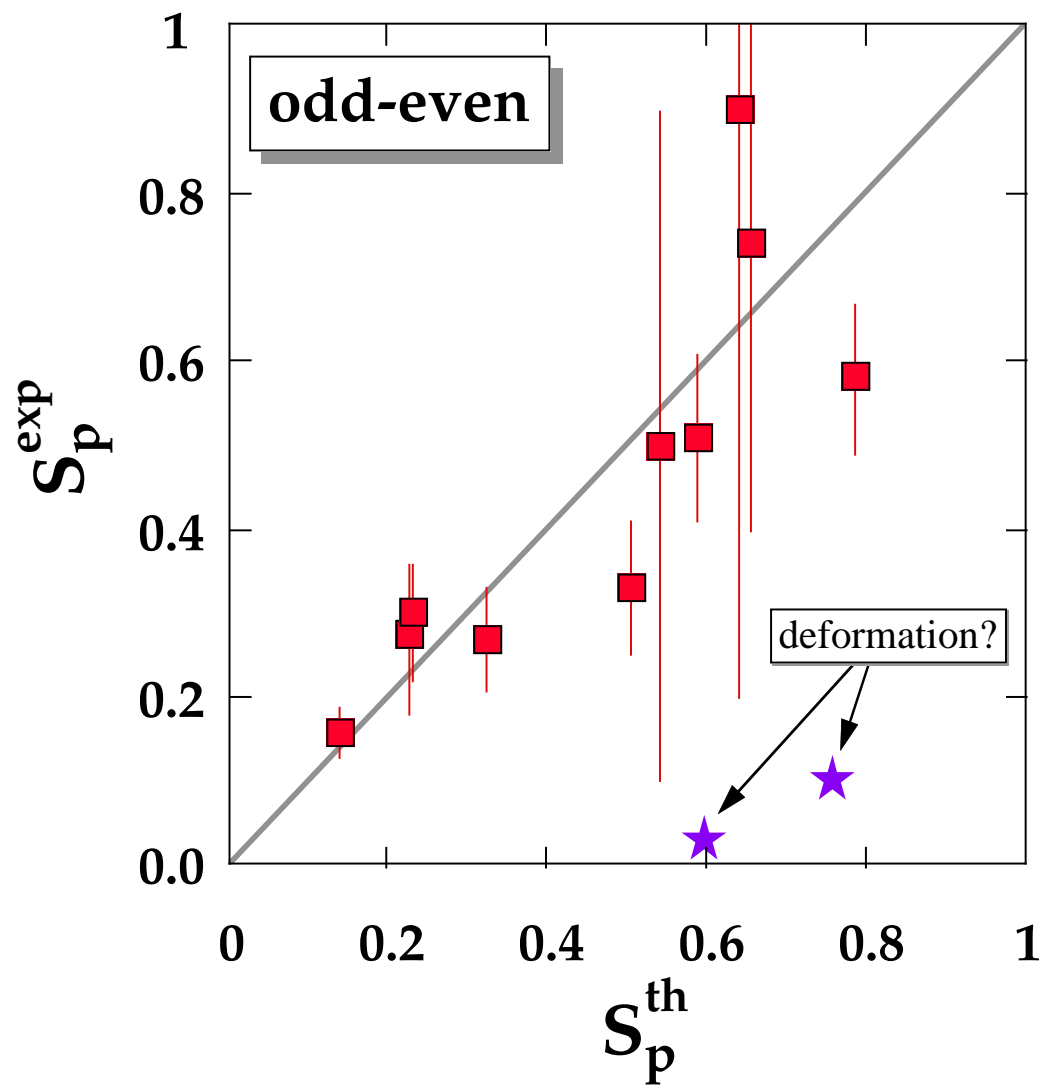

FIG. 11. Correlation between the experimental proton spectroscopic factors $S_{p}^{\exp }$ deduced from measured ground-state proton emission half-lives of odd- $Z$, even- $N$ proton emitters and theoretical values $S_{p}^{\text {th }}$ obtained in the BCS theory. The nuclei expected to be deformed are indicated by stars. (From Ref. 161.)

The correlation between experimental and theoretical spectroscopic factors obtained in the DWBA calculations is shown in Fig. 11 for odd- $Z$, even- $N$ ground-state proton emitters. The agreement between experiment and theory is good. For the two cases indicated by stars, 
${ }^{109} \mathrm{I}$ and ${ }^{113} \mathrm{Cs}$, the experimental values fall well below theoretical predictions. This suggests a strong fragmentation of the single-particle strength and/or increased tunneling probability as compared to spherical predictions. Indeed, both ${ }^{109} \mathrm{I}$ and ${ }^{113} \mathrm{Cs}$ are predicted to be deformed, and the "anomalous" proton half-life of ${ }^{109} \mathrm{I}$ has been reproduced by deformed calculations of Ref. 164.

In general, proton emission half-lives depend mainly on the proton separation energy and orbital angular momentum, but rather weakly on the details of intrinsic structure of proton emitters, e.g., on the parameters of the proton potential at least at a qualitative level (factors of 2-3). The weak sensitivity of $t_{1 / 2}$ to the details of the optical proton potential has been discussed in Ref. [46] in the context of two-proton radioactivity. It has been shown that more than 94-99\% of the WKB exponent comes from the region $r>r_{B}$, which is almost solely determined by the combined Coulomb and centrifugal potentials. This suggests that the lifetimes of deformed proton emitters will provide direct information on the angular momentum content of the associated Nilsson state, and hence, indirectly on the nuclear shape.

Proton radioactivity is an excellent example of the elementary three-dimensional quantum-mechanical tunneling. Experimental and theoretical investigations of proton emitters (or theoretically predicted ground-state di-proton emitters) will open up a wealth of exciting physics associated with the residual interaction coupling between bound states and extremely narrow resonances in the region of very low density of single-particle levels.

\section{CONCLUSIONS}

An experimental excursion into new territories of the chart of the nuclides will offer many excellent opportunities for traditional nuclear structure. This may include new regions of quadrupole and octupole deformation, new regions of shape isomers, including superdeformations, new combinations of magic or semi-magic closures, and many others. For a comprehensive review of these possibilities, the reader is referred to Ref. [165].

In trying to see the phenomena of a "new physics", we should ask the fundamental question of "how far is far"? Experiments with radioactive beams are going to be long and difficult, and many examples of nuclear exotica discussed in this paper (especially those concerning very neutron-rich systems) are clearly out of reach, even assuming most optimistic experimental scenarios. The hope is, however, that some of the effects associated with the loose binding will be seen as deviations from smooth systematic trends [91] or will show up at higher excitation energies closer to the particle threshold, as in the example of the analog states [126, 127]. Theoretically, we are bound to adopt the strategy of going to the extreme values of $N / Z$ in order to identify the qualitatively new phenomena, and then back down to experimentally achievable regions to see whether these phenomena can actually be observed. There is very little doubt that we are on the verge of the most fascinating fishing expedition; a lot of exciting physics will probably be caught already at the beginning of this journey.

The main objective of this study was to discuss various theoretical facets of nuclear structure with radioactive beams. In particular, the unusual conditions created by the weak binding and the importance of the coupling to the particle continuum have been emphasized. The theoretical formalism has been applied to experimental observables; i.e., energy spectra, 
masses, radii, surface thickness, and pair transfer form factors. It is demonstrated that these observables carry invaluable information that can pin down many basic questions regarding the effective nucleon-nucleon interaction.

The analysis presented in this paper should be viewed as a useful starting point for future investigations. One of them is the coupling between vibrational and rotational modes and pairing fields in weakly bound nuclei. Another interesting avenue of exploration is the role of dynamics; e.g., the importance of the particle number conservation and isospin mixing. A fascinating and difficult research program is the microscopic description of excited states, especially those lying above the particle emission threshold. We are only beginning to explore many unusual aspects of the nuclear many-body problem offered by systems with extreme $N / Z$ ratios.

\section{ACKNOWLEDGMENTS}

The Joint Institute for Heavy Ion Research has as member institutions the University of Tennessee, Vanderbilt University, and the Oak Ridge National Laboratory; it is supported by the members and by the Department of Energy through Contract No. DEFG05-87ER40361 with the University of Tennessee. This research was supported in part by the U.S. Department of Energy through Contract No. DE-FG02-96ER40963 with the University of Tennessee, and by the Polish Committee for Scientific Research under Contract No. 2 P03B 03408 with the Warsaw University. Oak Ridge National Laboratory is managed

for the U.S. Department of Energy by Lockheed Martin Energy Research Corporation under Contract No. DE-AC05-96OR22464. 


\section{REFERENCES}

[1] J. Dạbrowski, Nukleonika 22, 143 (1977).

[2] J. Freidrich and P.G. Reinhard, Phys. Rev. C33, 335 (1986).

[3] J.M. Pearson and M. Farine, Phys. Rev. C50, 185 (1994).

[4] M.M. Sharma, G. Lalazissis, G. König, and P. Ring, Phys. Rev. Lett. 74, 3744 (1995).

[5] P.-G. Reinhard and H. Flocard, Nucl. Phys. A584, 467 (1995).

[6] E. Chabanat, P. Bonche, P. Haensel, J. Meyer, and F. Schaeffer, Physica Scripta T56, 231 (1995).

[7] M. Onsi, R.C. Nayak, J.M. Pearson, H. Freyer, W. Stocker, Phys. Rev. C55, 3166 (1997).

[8] B.S. Pudliner, A. Smerzi, J. Carlson, V.R. Pandharipande, S.C. Pieper, and D.G. Ravenhall, Phys. Rev. Lett. 76, 2416 (1996).

[9] G.F. Bertsch and H. Esbensen, Ann. Phys. (N.Y.) 209, 327 (1991).

[10] J. Dobaczewski, W. Nazarewicz, T.R. Werner, J.-F. Berger, C.R. Chinn, and J. Dechargé, Phys. Rev. C53, 2809 (1996).

[11] S.A. Fayans and D. Zawischa, Phys. Lett. 383B, 19 (1996).

[12] Y. Shen and Z. Ren, Z. Phys. A356, 133 (1996).

[13] J. Dobaczewski, W. Nazarewicz, and T.R. Werner, Physica Scripta T56, 15 (1995).

[14] T.T. Kuo, F. Krmpotić, and Y. Tzeng, Phys. Rev. Lett. 78, 2708 (1997).

[15] J. Cugnon, P. Deneye and A. Lejeune, Z. Phys. A328, 409 (1987).

[16] G.E. Brown, Phys. Rep. 163, 167 (1988).

[17] S. Gmuca, Z. Phys. A342, 387 (1992).

[18] C.J. Pethick and D.G. Ravenhall, Annu. Rev. Nucl. Part. Sci. 45, 429 (1995).

[19] C.J. Pethick, D.G. Ravenhall, and C.P. Lorenz, Nucl. Phys. A584, 675 (1995).

[20] J. Dobaczewski, H. Flocard and J. Treiner, Nucl. Phys. A422, 103 (1984).

[21] U. Fano, Phys. Rev. 124, 1866 (1961).

[22] W. Glöckle, J. Hufner, and H.A. Weidenmueller, Nucl. Phys. A90, 481 (1967).

[23] R.H. Ibarra and B.F. Bayman, Phys. Rev. C1, 1786 (1970).

[24] R.J. Philpott, Fizika 9, suppl. 3, 21 (1977).

[25] H.W. Barz, I. Rotter, and J. Höhn, Nucl. Phys. A275, 111 (1977).

[26] M. Micklinghoff, Nucl. Phys. A295, 228 (1978).

[27] D. Halderson and R.J. Philpott, Nucl. Phys. A345, 141 (1980).

[28] W. Iskra and I. Rotter, Phys. Rev. C44, 721 (1991).

[29] W.M. Wendler, Nucl. Phys. A472, 26 (1987).

[30] M. Bolsterli, E.O. Fiset, J.R. Nix, and J.L. Norton, Phys. Rev. C5, 1050 (1972).

[31] W. Nazarewicz, T.R. Werner, and J. Dobaczewski, Phys. Rev. C50, 2860 (1994).

[32] J.R. Bennett, J. Engel, and S. Pittel, Phys. Lett. B368, 7 (1996).

[33] F. Ghielmetti, G. Colo, E. Vigezzi, P.F. Bortignon, and R.A. Broglia, Phys. Rev. C54, R2143 (1996).

[34] J.S. Vaagen, B.S. Nilsson, J. Bang, and R.M. Ibarra, Nucl. Phys. A319, 143 (1979).

[35] G. Rawitscher, Phys. Rev. C25, 2196 (1982).

[36] M. Buballa, S. Dróżdż, S. Krewald, and J. Speth, Ann. Phys. 208, 346 (1991).

[37] D. Ridikas, M.H. Smedberg, J.S. Vaagen, and M.V. Zhukov, Europhys. Lett. 37, 385 (1997).

[38] W.J. Romo, Nucl. Phys. A191, 65 (1972). 
[39] T. Berggren, Nucl. Phys. A109, 265 (1968).

[40] T. Vertse, P. Curutchet, and R.J. Liotta, Lecture Notes in Physics 325 (Springer Verlag, Berlin 1987), p. 179.

[41] P. Lind, R.J. Liotta, E. Maglione, and T. Vertse, Z. Phys. A347, 231 (1994).

[42] J.-F. Berger, L. Bitaud, J. Dechargé, M. Girod and S. Peru-Desenfants, Proc. of Int. Hirschegg Workshop XXIV, Extremes of Nuclear Structure edited by H. Feldmeier, J. Knoll, and W. Nörenberg, (GSI, Darmstadt, 1996), p. 56.

[43] S. Fortunato, A. Insolia, R.J. Liotta, and T. Vertse, Phys. Rev. C54, 3279 (1997).

[44] N. Sandulescu, R.J. Liotta, and R. Wyss, Phys. Lett. B394, 6 (1997).

[45] J. Dobaczewski, W. Nazarewicz, and T.R. Werner, Z. Phys. A354, 27 (1996).

[46] W. Nazarewicz, J. Dobaczewski, T.R. Werner, J.A. Maruhn, P.-G. Reinhard, K. Rutz, C.R. Chinn, A.S. Umar, and M.R. Strayer, Phys. Rev. C53, 740 (1996).

[47] J. Terasaki, P.-H. Heenen, H. Flocard, and P. Bonche, Nucl. Phys. A600, 371 (1996).

[48] K. Mühlhans, K. Neergård, and U. Mosel, Nucl. Phys. A420, 204 (1984).

[49] P.-G. Reinhard, M. Bender, K. Rutz, and J.A. Maruhn, Preprint nucl-th/9705054.

[50] V.A. Khodel' and É.E. Sapershtel̆, Phys. Rep. 92, 12 (1982).

[51] M.V. Zverev and É.E. Sapershteŭn, Sov. J. Nucl. Phys. 39, 878 (1984).

[52] M.V. Zverev and É.E. Sapershteı̆n, Sov. J. Nucl. Phys. 42, 683 (1985).

[53] A.V. Smirnov, S.V. Tolokonnikov, and S.A. Fayans, Sov. J. Nucl. Phys. 48, 995 (1988).

[54] M.V. Zverev and V.E. Starodubsky, Sov. J. Nucl. Phys. 54, 410 (1991).

[55] J. Meng and P. Ring, Phys. Rev. Lett. 77, 3963 (1996).

[56] W. Poschl, D. Vretenar, P. Ring, Comp. Phys. Commun. 103, 217 (1997).

[57] A.B. Migdal, Theory of Finite Fermi Systems and Applications to Atomic Nuclei (Interscience, New York, 1967).

[58] S. Shlomo and G. Bertsch, Nucl. Phys. A243, 507 (1975).

[59] S. Kamerdzhiev, J. Speth, G. Tertychny, and J. Wambach, Z. Phys. A346, 253 (1993).

[60] H. Sagawa and C.A. Bertulani, Prog. Theor. Phys. (suppl.) 124, 143 (1996).

[61] I. Hamamoto, H. Sagawa, and X.Z. Zhang, Phys. Rev. C53, 765 (1996).

[62] I. Hamamoto and H. Sagawa, Phys. Rev. C53, R1492 (1996).

[63] I.N. Borzov, A.A. Fayans, and E.L. Trykov, Nucl. Phys. A584, 335 (1995).

[64] I.N. Borzov, A.A. Fayans, E. Krömer, and D. Zawischa, Z. Phys. A355, 117 (1996).

[65] J. Dobaczewski, I. Hamamoto, W. Nazarewicz, and J.A. Sheikh, Phys. Rev. Lett. 72 , 981 (1994).

[66] J. Dechargé and D. Gogny, Phys. Rev. C21, 1568 (1980).

[67] E.G. Nadjakov, K.P. Marinova, and Yu.P. Gangrsky, At. Data Nucl. Data Tables 56, 133 (1994).

[68] C.J. Batty, E. Friedman, H.J. Gills, and H. Rebel, Adv. Nucl. Phys. 19, 1 (1989).

[69] K. Riisager, A.S. Jensen, and P. Møller, Nucl. Phys. A548, 393 (1992).

[70] A.M. Lane, Nuclear Theory (Benjamin, New York, 1964).

[71] K.A. Brueckner, T. Soda, P. W. Anderson, and P. Morel, Phys. Rev. 118, 1442 (1960).

[72] V.J. Emery and A.M. Sessler, Phys. Rev. 119, 248 (1960).

[73] D.S. Delion, M. Baldo, and U. Lombardo, Nucl. Phys. A593, 151 (1995).

[74] H. Kucharek and P. Ping, Z. Phys. A339, 23 (1991).

[75] S.G. Kadmenskiü, P.A. Luk'yanovich, Yu. I. Remesov, and V.I. Furman, Sov. J. Nucl. Phys. 45, 585 (1987). 
[76] É.E. Sapershteĭn and M.A. Troitskiǔ, Sov. J. Nucl. Phys. 1, 284 (1965).

[77] D. Zawischa, U. Regge, and R. Stapel, Phys. Lett. 185B, 299 (1987).

[78] U. Regge and D. Zawischa, Phys. Rev. Lett. 61, 149 (1988).

[79] S.A. Fayans, S.V. Tolokonnikov, E.L. Trykov, and D. Zawischa, Phys. Lett. 338B, 1 (1994).

[80] A. Bulgac, Preprint FT-194-1980, Central Institute of Physics, Bucharest, 1980.

[81] S.T. Belyaev, A.V. Smirnov, S.V. Tolokonnikov, and S.A. Fayans, Sov. J. Nucl. Phys. 45, 783 (1987).

[82] B.E. Vonderfecht, C.C. Gearhart, W.H. Dickhoff, A. Polls, and A. Ramos, Phys. Lett. B253, 1 (1991).

[83] F. De Blasio and G. Lazzari, Nuovo Cimento 107A, 1549 (1994).

[84] F. Tondeur, Z. Phys. A288, 97 (1978).

[85] P. Haensel, J.L. Zdunik, and J. Dobaczewski, Astron. Astrophys. 222, 353 (1989).

[86] R. Smolańczuk and J. Dobaczewski, Phys. Rev. C48, R2166 (1993).

[87] B. Chen, J. Dobaczewski, K.-L. Kratz, K. Langanke, B. Pfeiffer, F.-K. Thielmann, and P. Vogel, Phys. Lett. B355, 37 (1995).

[88] J.M. Pearson, R.C. Nayak, and S. Goriely, Phys. Lett. B387, 455 (1996).

[89] E. Chabanat, Interactions effectives pour des conditions extrêmes d'isospin, Université Claude Bernard Lyon-1, Thesis 1995, LYCEN T 9501, unpublished.

[90] Z. Patyk, A. Baran, J.F. Berger, J. Dechargé, J. Dobaczewski, R. Smolańczuk, and A. Sobiczewski, Acta Phys. Pol. B27, 457 (1996).

[91] W.-T. Chou, R.F. Casten, and N.V. Zamfir, Phys. Rev. C51, 2444 (1995).

[92] C. Détraz, D. Guillemaud, G. Huber, R. Klapish, M. Langevin, F. Naulin, C. Thibault, L.C. Carraz, and F. Touchard, Phys. Rev. C19, 164 (1979).

[93] F. Touchard, J. M. Serre, S. Büttenbach, P. Guimbal, R. Klapisch, M. de Saint Simon, C. Thibault, H. T. Duong, P. Juncar, S. Libermen, J. Pinard, and J. L. Vialle, Phys. Rev. C25, 2756 (1982).

[94] H. Scheit, T. Glasmacher, B.A. Brown, J.A. Brown, P.D. Cottle, P.G. Hansen, R. Harkewicz, M. Hellstrom, R.W. Ibbotson, J.K. Jewell, K.W. Kemper, D.J. Morrissey, M. Steiner, P. Thirolf, and M. Thoennessen, Phys. Rev. Lett. 77, 3967 (1996).

[95] T. Glasmacher, B.A. Brown, M.J. Chromik, P.D. Cottle, M. Fauerbach, R.W. Ibbotson, K.W. Kemper, D.J. Morrissey, H. Scheit, D.W. Sklenicka, and M. Steiner, Preprint MSUCL-1048, 1977.

[96] X. Campi, H. Flocard, A. K. Kerman, and S. Koonin, Nucl. Phys. A251, 193 (1975).

[97] T.R. Werner, J.A. Sheikh, W. Nazarewicz, M.R. Strayer, A.S. Umar, and M. Misu, Phys. Lett. B333, 303 (1994).

[98] G. Audi and A.H. Wapstra, Nucl. Phys. A565, 1 (1993); Nucl. Phys. A565, 66 (1993).

[99] K.-L. Kratz, J.-P. Bitouzet, F.-K. Thielemann, P. Möller, and B. Pfeiffer, Astrophys. J. 403, 216 (1993).

[100] W.M. Howard, S. Goriely, M. Rayet, and M. Arnould, Astrophys. J. 417, 713 (1993).

[101] B. Pfeiffer, K.-L. Kratz, and F.-K. Thielemann, Z. Phys. A357, 235 (1997).

[102] R. Surman, J. Engel, J.R. Bennett, and B.S. Meyer, Preprint astro-ph/9701007.

[103] S. Goriely and M. Arnould, Astron. Astrophys. 312, 327 (1996).

[104] W.C. Haxton, K. Langanke, Y.Z. Qian, and P. Vogel, Phys. Rev. Lett. 78, 2694 (1997).

[105] Y.Z. Qian, W.C. Haxton, K. Langanke, and P. Vogel, Phys. Rev. C55, 1532 (1997). 
[106] A. Staudt, E. Bender, K. Muto, and H.V. Klapdor, Z. Phys. A334, 47 (1989).

[107] P. Möller and J. Randrup, Nucl. Phys. A514, 1 (1990).

[108] T. Tachibana and M. Arnould, Nucl. Phys. A588, 333c (1995).

[109] E.P. Wigner, Phys. Rev. 73, 1002 (1948).

[110] T. Uchiyama and H. Morinaga, Z. Phys. A320, 273 (1985).

[111] S.A. Fayans, Phys. Lett. B267, 443 (1991).

[112] M. Yokoyama, T. Otsuka, and N. Fukunishi, Phys. Rev. C52, 1122 (1995).

[113] H. Sagawa, N. Van Giai, N. Takigawa, M. Ishihara, and K. Yazaki, Z. Phys. A351, 385 (1995).

[114] M. Fukuda, T. Ichihara, N. Inabe, T. Kubo, H. Kumagai, T. Nakagawa, Y. Yano, I. Tanihata, M. Adachi, K. Asahi, M. Kouguchi, M. Ishihara, H. Sagawa, and S. Shimoura, Phys. Lett. B 268, 339 (1991).

[115] S. Raman, C.H. Malarkey, W.T. Milner, C.W. Nestor, Jr., and P.H. Stelson, Atomic Data Nucl. Data Tables 36, 1 (1987).

[116] M.Bouten, E. Flerackers, M.C. Bouten, Nucl. Phys. A307, 413 (1978).

[117] I. Ragnarsson, S. Åberg, H.-B. Håkansson and R.K. Sheline, Nucl. Phys. A361, 1 (1981).

[118] Y. Kanada-Enyo, H. Horiuchi, A. Ono, Phys. Rev. C52, 628 (1995).

[119] H. Esbensen, B.A. Brown, H. Sagawa, Phys. Rev. C51, 1274 (1995).

[120] N. Vinh Mau, Nucl. Phys. A592, 33 (1995).

[121] C.A. Bertulani, H. Sagawa, Nucl. Phys. A588, 667 (1995).

[122] X. Li and P.-H. Heenen, Phys. Rev. C54, 1617 (1996).

[123] T. Misu, W. Nazarewicz, and S. Åberg, Nucl. Phys. A614, 44 (1997).

[124] T.R. Werner, J.A. Sheikh, M. Misu, W. Nazarewicz, J. Rikovska, K. Heeger, A.S. Umar, and M.R. Strayer, Nucl. Phys. A597, 327 (1996).

[125] F. Naulin, P. Bonche, H. Flocard, and P.H. Heenen, Abstract at the Conference on Nuclear Structure at the Limits, Argonne 1996, ANL/PHY-96/1, p. 143.

[126] R.G. Thomas, Phys. Rev. 81, 148 (1951); 88, 1109 (1952).

[127] J.B. Ehrman, Phys. Rev. 81, 412 (1951).

[128] S. Schlomo, Rep. Prog. Phys. 41, 957 (1978).

[129] R. Schneider, J. Friese, J. Reinhold, K. Zeitelhack, T. Faestermann, R. Gernhauser, H. Gilg, F. Heine, J. Homolka, P. Kienle, H.J. Körner, H. Geissel, G. Münzenberg, and K.Summerer, Z. Phys. A 348, 241 (1994).

[130] M. Lewitowicz, R. Anne, G. Auger, D. Bazin, C. Borcea, V. Borrel, J.M. Corre, T. Dorfler, A. Fomichov, R. Grzywacz, D. Guillemaud-Mueller, R. Hue, M. Huyse, Z. Janas, H. Keller, S. Lukyanov, A.C. Mueller, Yu. Penionzhkevich, M. Pfutzner, F. Pougheon, K.Rykaczewski, M.G. Saint-Laurent, K. Schmidt, W.D. Schmidt-Ott, O. Sorlin, J. Szerypo, O. Tarasov, J. Wauters, and J. Żylicz, Phys. Lett. B332, 20 (1994).

[131] M. Chartier, G. Auger, W. Mittig, A. Lepine-Szily, L.K. Fifield, J.M. Casandjian, M. Chabert, J. Ferme, A. Gillibert, M. Lewitowicz, M. Mac Cormick, M.H. Moscatello, O.H. Odland, N.A. Orr, G. Politi, C. Spitaels, and A.C.C. Villari, Phys. Rev. Lett. 77, 2400 (1996).

[132] A. Goswami, Nucl. Phys. 60, 228 (1964).

[133] A. Goswami and L.S. Kisslinger, Phys. Rev. 140 , B26 (1965).

[134] P. Camiz, A. Covello, and M. Jean, Nouvo Cimento 36, 663 (1965); 42B, 199 (1966). 
[135] J. Bar-Touv, A. Gosvami, A.L. Goodman, and G.L. Struble, Phys. Rev. 178, 178 (1969).

[136] H.T. Chen, H. Müther, and A. Faessler, Nucl. Phys. A297, 445 (1978).

[137] W. Nazarewicz and S. Pittel, http://aps.org/BAPSAPR97/vpr/laye8.htm.

[138] A.L. Goodman, Adv. Nucl. Phys. 11, 263 (1979).

[139] N. Zeldes, in Handbook of Nuclear Properties, Ed. by D. Poenaru and W. Greiner, Clarendon Press, Oxford, 1996, p. 13.

[140] J.M.C. Chen, J.W. Clark, R.D. Davé, and V.V. Khodel, Nucl. Phys. A555, 59 (1993).

[141] M.K. Cheoun, A. Bobyk, A. Faessler, F. Simkovic, and G. Teneva, Nucl. Phys. A561, 74 (1993).

[142] J. Schwieger, F. Simkovic, and A. Faessler, Nucl. Phys. A600, 179 (1996).

[143] J. Engel, S. Pittel, M. Stoitsov, P. Vogel, and J. Dukelsky, Phys. Rev. C55, 1781 (1997).

[144] P. Fröbrich, Z. Phys. 236, 153 (1970).

[145] P. Fröbrich, Phys. Lett. 37B, 338 (1971).

[146] J. Kvasil, A.K. Jain, and R.K. Sheline, Czech. J. Phys. 40, 278 (1990).

[147] W. Satuła and R. Wyss, Phys. Lett. B393, 1 (1997).

[148] F. Palumbo, Lett. Nuovo Cimento 14, 572 (1975).

[149] T. Alm, G. Röpke, and M. Schmidt, Z. Phys. 337, 355 (1990).

[150] Y. Aboussir, J.M. Pearson, A.K. Dutta, and F. Tondeur, At. Data Nucl. Data Tables 61, 127 (1995).

[151] W. Satuła, D.J. Dean, J. Gary, S. Mizutori, and W. Nazarewicz, Phys. Lett. B, in press.

[152] D.S. Brenner, C. Wesselborg, R.F. Casten, D.D. Warner and J.-Y. Zhang, Phys. Lett. 243B, 1 (1990).

[153] J. Engel, K. Langanke, and P. Vogel, Phys. Lett. B389, 211 (1996).

[154] E. Perlińska, S.G. Rohoziński, J. Dobaczewski, and W. Nazarewicz, Proc. of Int. Hirschegg Workshop XXIV, Extremes of Nuclear Structure edited by H. Feldmeier, J. Knoll, and W. Nörenberg, (GSI, Darmstadt, 1996), p. 228.

[155] A.L. Goodman, Nucl. Phys. A186, 475 (1972).

[156] H. Wolter, A. Faessler, and P. Sauer, Phys. Lett. 31B, 516 (1970); Nucl. Phys. A167, 108 (1971).

[157] S. Hofmann, Radiochim. Acta 70/71, 93 (1995).

[158] S. Hofmann, in: Nuclear Decay Modes, ed. by D.N. Poenaru and W. Greiner, (IOP, Bristol, 1996); Preprint GSI-93-04, 1993.

[159] S.G. Kadmenskiu and V.E. Kalechtis, Sov. J. Nucl. Phys. 12, 37 (1971).

[160] W.F. Feix and E.R. Hilf, Phys. Lett. 120B, 14 (1983).

[161] S. Åberg, P.B. Semmes, and W. Nazarewicz, Phys. Rev. C, October 1997.

[162] S.A. Gurvitz, Phys. Rev. A38, 1747 (1988).

[163] B. Buck, A.C. Merchant, and S.M. Perez, Phys. Rev. C45, 1688 (1992).

[164] S.G. Kadmenskiǐ and V.P. Bugrov, Phys. Atomic Nuclei 59, 399 (1996).

[165] The IsoSpin Laboratory: Research opportunities with Radioactive Nuclear Beams, Preprint LALP 91-51. 